\title{
PROPOSTA EDUCATIVA COM ENFOQUE ERGONÔMICO PARA AUXILIAR NA PREVENÇÃO DE LESÕES MÚSCULO-ESQUELÉTICAS NA EQUIPE DE ENFERMAGEM
}

\author{
EDUCATIONAL PROPOSAL FOCUSED ON ERGONOMICS AS PREVENTION TOOL \\ AGAINST MUSCULUSKELETAL INJURIES FOR NURSING STAFF \\ PROPUESTA EDUCATINA CON ENFOQUE ERGONÓMICO PARA AUXILIAR EN LA \\ PREVENCIÓN DE LESIONES MÚSCULO-ESQUELÉTICAS EN \\ EQUIPO DE ENFERMERÍA
}

\section{Neusa Maria Costa Alexandre 2 Marco Antonio Alves de Moraes ${ }^{3}$}

RESUMO: Os trabalhadores de enfermagem estão sujeitos a lesões na coluna vertebral, atribuídas a condições ergonômicas e posturais inadequadas. Com o objetivo de auxiliar na redução dessas lesões, elaborou-se uma proposta educativa específica para o pessoal de enfermagem, cujo planejamento e tópicos constam do presente estudo. A estratégia foi realizada a partir da análise do ambiente de trabalho e das atividades desenvolvidas por esse pessoal, abordando temas relativos à anatomia e biomecânica da coluna vertebral, orientações gerais de postura, aspectos ergonômicos específicos do trabalho da enfermagem e considerações sobre movimentação e transporte de pacientes.

PALAVRAS CHAVE: Dores nas costas, Ergonomia, Educação em saúde

ABSTRACT: Nursing staff is subject to lesions in the back due to ergonomic factors and inadequate posture. A specific educational proposal for nursing personnel was elaborated with the objective of helping to reduce these lesions. This study presents the development and topics of this educational strategy that was constructed from an analysis of the work environment and of the activities engaged in by this personnel. Themes relative to the anatomy and biomechanics of the vertebral column were presented, as well as general posture orientations, specific ergonomic aspects of nursing tasks and considerations about the movement and transportation of patients.

KEYWORDS: Back pain, Ergonomics, Health education

RESUMEN: El equipo de enfermería es propenso a lesiones en la columna vertebral, atribuídas a condiciones ergonómicas y posturales inadecuadas. Con el objetivo de auxiliar en la redicción de estas lesiones, se elaboró una propuesta educativa específica para el personal de enfermería, cuyo planeamento y tópicos constan en el presente estudio. La estrategia fue realazada a partir del análisis del ambiente de trabajo y de las actividades desempeñadas por esse personal, abordando temas relativos a anatomia y biomecánica de la culumna vertebral, orientaciones generales de postura, aspectos ergonómicos y transporte de pacientes.

PALABRAS CLAVE: Dolor de espalda, Ergonomia, Educación en salud

\footnotetext{
1 Apresentado no 49ํㅡㄴ congresso Brasileiro de Enfermagem-Belo Horizonte-MG, dezembro de 1997.

${ }^{2}$ Enfermeira. Prof. Assistente Doutor do Departamento de Enfermagem da Faculdade de Ciências Médicas- UNICAMP.

${ }^{3}$ Fisioterapeuta do centro de saúde da comunidade da UNICAMP. Pós-graduando da Faculdade de Educação Física- UNICAMP.
} 


\section{INTRODUÇÃO}

As lesões do sistema músculo-esquelético têm despertado a atenção de pesquisadores preocupados com questões relativas à saúde e trabalho, visto representarem as principais causas de incapacitação de adultos, e também pelo impacto provocado nas esferas psicossociais e econômicas, (Parniapour et al. 1990, Yeliu; Callahan, 1995). Entre essas afecções incluem-se as enfermidades da coluna vertebral que constituem um complexo desafio para a Saúde Ocupacional.

Pesquisadores e organizações de várias partes do mundo têm destacado a equipe de enfermagem como um grupo de risco em relação ao desenvolvimento de dores osteomusculares, particularmente as algias vertebrais. (Alexandre, 1993, Burgmeier, 1988, Cohen - Mansfield, 1996, Engles, 1996, Guo, 1995, Harber, 1985, Knibbe; Friele, 1996, Leighton; Reilly, 1995, Stubbs, 1983, Videman, 1984, WORLD HEALTH ORGANIZATION, 1985, Yassi et al., 1995). Um relevante aspecto dos problemas osteomusculares é a questão da etiologia, pois são causados por inúmeros fatores inter-relacionados (WORLD HEALTH ORGANIZATION, 1985). Geralmente consideram-se os fatores de risco como individuais e relacionados com o ambiente de trabalho, sendo mais citados os defeitos posturais e congênitos, força muscular, trabalho físico pesado, levantamento e manuseio de cargas, manutenção de uma postura por tempo prolongado, movimentos freqüentes de flexão e torção da coluna vertebral, entre outros. Recentemente estão sendo ampliados para aspectos como condições socio-econômicas, fatores psicológicos, vida sedentária e a própria organização do trabalho. (Barreira, 1989, Garg; Moore, 1992, Magui et al., 1994, Parniapour et al., 1990, Ross, 1994, Svenson; Handerson, 1989, WORLD HEALTHORGANIZATION, 1985).

Inúmeros pesquisadores apresentam estratégias para tentar a solução de problemas relacionados ao sistema músculo-esquelético. A revisão da literatura aponta abordagens tais como: treinamento, administração de cursos sobre mecânica corporal, programa de exercícios e alongamentos, mudanças no ambiente e posto de trabalho, alteração do estilo de vida, entre outros. (Chaffin, 1987, De Rosa; Porterfield, 1992, Douchin, 1990, Kellet, 1991, Leonard, 1990, Linton, 1989, Moffett et al., 1986, Reilly et al., 1989, Westgmrd, 1985).

Desenvolve-se aqui uma estratégica específica para a equipe de enfermagem de um hospital universitário, através de uma abordagem educativa, partindo da análise do ambiente de trabalho e atividades desenvolvidas por estes profissionais. Trata de temas relativos à anatomia e biomecânica da coluna vertebral, com orientação geral de postura, aspectos ergonômicos específicos dos serviços e consideração sobre movimentação e transporte de pacientes. O presente estudo tem por objetivo apresentar o planejamento e tópicos dessa abordagem educativa.

\section{PLANEJAMENTO DA ESTRATÉGIA EDUCATIVA}

Grande parte das agressões à coluna vertebral na equipe de enfermagem estão relacionadas a condições ergonômicas inadequadas de mobiliários, posto de trabalho e equipamentos utilizados nas atividades cotidianas da enfermagem, sendo as dores nas costas causadas por traumas crônicos repetitivos, que envolvem 
muitos outros fatores, além da manipulação de pacientes. (Alexandre, 1993, Harber, 1987, Margiale, 1995, UNIVERSITY OF SURREY, 1986). Muitos autores têm sugerido a administração de cursos sobre movimentação e transporte de pacientes como uma forma de reduzir a incidência de problemas na coluna vertebral entre os trabalhadores de enfermagem. (Daws, 1981, Raistrick, 1981, Scholey, 1984, Thompson, 1987). A utilização de auxílios mecânicos também tem sido indicada para prevenir as dores nas costas. (Hill, 1990, Lenihan, 1983).

Atualmente sabe-se que para resolver tais problemas é necessário um amplo estudo do ambiente, equipamentos e dos indivíduos, utilizando um enfoque ergonômico (Stubbs, 1983, UNIVERSITY OF SURREY, 1986). Assim, as habilidades em movimentação de pacientes devem ser complementadas com o estabelecimento de práticas seguras de trabalho dentro de uma estrutura ergonômica.

Pesquisas voltadas para o enfoque de programas instrucionais que envolvem princípios de ergonomia e biomecânica têm mostrado que este treinamento representa uma das estratégias para auxiliar a prevenção de lesões dorsais. (Linton et al., 1989, Troup; Rauhala, 1987, Venning, 1988, Videman, 1989). A abordagem educativa aqui apresentada foi desenvolvida tendo como subsídios teóricos alguns estudos internacionais e pesquisas ergonômicas realizadas no hospital universitário utilizado. (Alexandre, 1987, Alexandre, 1992, Alexandre, 1993, Troup; Rauhala, 1987, Venning, 1988). Assim, planejou-se um curso a partir da análise do ambiente de trabalho e das atividades aí desenvolvidas especificamente pelo pessoal de enfermagem.

\section{DESCRIÇÃO DA PROPOSTA EDUCATIVA}

Foi dividida em seis diferentes e independentes módulos que deverão ser apresentados paulatinamente no decorrer do programa de exercícios:

1. Dados fundamentais da anatomia da coluna vertebral

2. Mecânica corporal

3. Situações que podem causar dores nas costas

4. Orientações ergonômicas específicas

5. Orientações gerais

6. Movimentação e transporte de pacientes

Esses módulos serão apresentados separadamente a seguir:

\section{DADOS FUNDAMENTAIS DA ANATOMIA DA COLUNA VERTEBRAL}

O conhecimento das estruturas anatômicas que compõem a coluna mesmo tempo articulada e flexível, por ser formada por múltiplas peças sobrepostas, unidas umas às outras por elementos ligamentares e musculares. É composta pela superposição de 33 vértebras, separadas estruturalmente em cinco regiões, que de cima para baixo são: 7 cervicais, 12 torácicas, 5 lombares e 9 sacrococcígeas fundidas. Uma visão anterior ou posterior revela uma coluna vertebral alinhada, contrariamente à vista lateral em que se observam 4 curvaturas, que são de cima para baixo: a lordose cervical, com convexidade anterior e a curvatura sacroccígea, que se apresenta fixa e com convexidade posterior .(Hall, 1993, Kapandji, 1979). 
Anatomicamente, a unidade básica da coluna vertebral compreende um segmento móvel que é constituído por duas porções: uma anterior, formada pelos corpos vertebrais e um disco intervertebral, e uma posterior, que contém as facetas articulares, músculos e ligamentos.

Neste segmento posterior observa-se também o canal medular, que tem início no forâmen occipital, alojando o bulbo e a medula espinhal, representando assim um protetor elástico e eficaz deste eixo nervoso. A unidade compreende também os tecidos muscular e aponeurótico adjacentes, artérias, veias e tecido adiposo. Apoiando-se sobre uma base pélvica móvel, a estrutura óssea da coluna vertebral representa a parte rígida, enquanto as formações ligamentosas são elementos de reforço e estabilização. Os grupos musculares desempenham importante papel na sustentação e manutenção da coluna vertebral, permitindo ao mesmo tempo a sua movimentação. (Hall, 1993, Patenaude; Sommer, 1987).

A anatomia da coluna vertebral é assim apresentada por meio de "slides", de acordo com a seqüência descrita acima.

\section{MECÂNICA CORPORAL}

A maneira cuidadosa e eficiente de usar todas as partes do corpo em relação ás forças internas e externas contribui para evitar possíveis agressões ao sistema músculo esquelético, especialmente à coluna vertebral. Para abranger as necessidades músculo- esqueléticas do paciente e a do próprio trabalhador enquanto sujeito ativo do movimento, os profissionais de enfermagem devem compreender e utilizar de forma adequada os princípios de mecânica corporal. Nessa fase são abordados temas como postura, gravidade, equilíbrio, disco intervertebral e biomecânica da coluna vertebral, que são descritos a seguir.

Um dos primeiros passos para compreensão da mecânica corporal é considerar a postura. No homem, a postura ereta é organizada por limitada e selecionada combinação de posições de diferentes segmentos corporais. Para a postura convergem todos os elementos que caracterizam o movimento, sendo ela mesma a base de cada atividade cinética e a resultante da integridade das diversas estruturas anatômicas e seu funcionamento. Muitos autores e entidades tentam definir o que é postura.

Para Kendall et al. (1977) é a posição corporal que envolve o mínimo de estreitamento e de 'stress" das estruturas, com o menor gasto de energia para se obter o máximo de eficiência no uso do corpo. Para estudar os movimentos humanos, que são modificações da postura estática, é importante conhecer o centro de gravidade do corpo que, para Jensen e Schultz (1979), pode ser definido como: o ponto exato em que o corpo poderia teoricamente, ser rodado livremente em todas as direções; o centro em torno do qual o corpo deveria ter o mesmo peso e o ponto de interseção dos seus três planos cardinais, o sagital, o frontal e o transverso.

Existe a aceitação geral de que uma postura adequada é aquela em que a linha de gravidade (considerada uma linha vertical através do centro de gravidade) passa pelos seguintes. pontos anatômicos: apófise mastóide, extremidade lateral. 0 equilíbrio de um corpo é obtido quando está em repouso ou movimentando-se em contrabalanço de um outro conjunto de forças ou de movimentos. Podem ser 
deduzidos vários pontos básicos: que um objeto é mais estável se tem uma ampla base de apoio, se o seu centro de gravidade está próximo desta base e se a linha de gravidade passa através da mesma. A postura corporal ereta é obtida pelo equilíbrio entre as forças que agem no centro de gravidade, puxando o corpo para o solo e a força dos músculos antigravitacionais, que tracionam em sentido contrário. Se os músculos falharem, o corpo colapsará em forma de flexão, pela ação da força da gravidade. Assim, a maioria dos músculos antigravitacionais são os extensores, principalmente do pescoço, das costas e das pernas. (Hall, 1993).

Para que se mantenha em condições normais a atividade muscular, que permite movimento e sustentação, há necessidade de períodos semelhantes de contração e relaxamento. A atividade muscular estática por tempo prolongado pode provocar o aparecimento de processos irritativos e inflamatórios nas estruturas osteomusculares, em especial nos músculos, e conduzir a quadros de tensão, contratura e dor.

Apoiada em todos estes dados, a coluna vertebral, eixo do corpo, precisa conciliar dois imperativos mecânicos contraditórios: a rigidez e a elasticidade. Isto se deve a sua estrutura fixa e constituição por múltiplas peças sobrepostas, unidas umas às outras por elementos ligamentares e musculares. Como uma permite movimentos nos três planos do espaço: sendo pequena a movimentação entre vértebras adjacentes, a anormalidade em uma das três deverá afetar as outras duas. As facetas articulares guiam e limitam os movimentos do segmento móvel, restringindo a movimentação anterior da vértebra superior sobre a inferior.

Entre cada corpo vertebral encontra-se uma estrutura importante da coluna, denominada disco intervertebral, cuja função primária é ligar as unidades vertebrais, mantendo-as flexíveis, atuando também como amortecedor de choque. Seuenvoltório externo ( anel fibroso ) é rígido e forte, contendo as forças contrárias exercidas pelas vértebras. O núcleo central (núcleo pulposo) apresenta elevado teor aquoso, com propriedades viscosas, que sob pressão exerce tensão em todas as direções. Juntos, o anel fibroso e o núcleo pulposo compõem uma estrutura balanceada. Em uma pessoa jovem, o disco intervertebral compreende aproximadamente $20 \%$ do comprimento da coluna vertebral. Com mudanças degenerativas e menor volume de disco, esta porcentagem é reduzida e a pessoa perde altura, principalmente no final do dia, quando pressões mecânicas causam a desidratação daquela estrutura. Sendo o disco adulto avascular, seu tecido depende de meios mecânicos para manter suas necessidades hidrófilas, de oxigênio, substâncias nutrientes e remoção dos produtos de resíduos. Acredita-se que a mobilidade espinhal promova e preserve a integridade do disco através de meios de descompressão mecânica.

Com o envelhecimento, o núcleo perde sua capacidade de fixar água, ocorrendo um decréscimo adicional das propriedades de osmose e absorção e, após a Segunda década de vida, desaparece a vascularização do disco intervertebral. $\mathrm{Na}$ terceira década, o disco é nutrido pela difusão de linfa através das placas terminais das vértebras e em virtude das características físico-químicas de absorção do gel coloidal no núcleo. Desta forma, a altura de um disco lesado deve estar 
diminuída, comprometendo sua capacidade de amortecimento e as suas relações articulares inter-apofisárias das vértebras, podendo representar um fator de artrose. (Kapandji, 1979, Knoplick, 1986, Patenaude; Sommer, 1987).

A solicitação repetitiva e muitas vezes extraordinárias imposta à coluna vertebral com maior freqüência são os esforços em flexão, para levantamento e transporte manual de cargas pesadas; esforço, para manipulação física ou trabalho físico intenso de empurrar, puxar ou empilhar objetos pesados; esforços inadequados, como as rotações de tronco sobre a pélvis, agravadas muitas vezes ao levantamento de cargas, inclinações laterais e flexão e extensão de tronco. Os esforços de compressão sobre o disco são tão mais intensos quanto mais próximo estiver do sacro, visto que o peso do corpo suportado aumenta com a altura suprajacente.

O conteúdo deste tópico é retomado nos próximos módulos, no relacionamento das orientações práticas com essa fundamentação teórica.

\section{SITUAÇÕES QUE PODEM CAUSAR DORES NAS COSTAS}

A literatura tem apresentado inúmeros fatores que contribuem para 0 aparecimento de lesões dorsais em trabalhadores de enfermagem (Alexandre, 1993, Harber, 1985, Marziale, 1995, UNIVERSITY OF SURREY, 1986, WORLD HEALTH ORGANIZATION, 1985). É importante ressaltar que esses fatores estão relacionados com uma variedade de aspectos associados ao paciente, equipamentos, posto de trabalho, atividades ocupacionais e ao próprio pessoal de enfermagem, o que inclui suas habilidades e capacidades psicofisiológicas e antropométricas.

Com base em publicações e em estudos realizados no hospital, foi possível fotografar as principais atividades e fatores ergonômicos relacionados com lesões no sistema músculo-esquelético, envolvendo o pessoal de enfermagem na sua própria unidade de serviço. Nessa fase do curso apresenta-se uma série de "slides" que procuram abordar determinadas situações problemas, com o objetivo de auxiliar no desenvolvimento de uma consciência crítica em relação aos efeitos do ambiente de trabalho sobre a saúde da equipe de enfermagem.

A apresentação é dividida em duas partes, sendo que a primeira enfoca as principais condições causadoras de problemas osteomusculares e a Segunda aborda os aspectos ergonômicos relacionados com o ambiente e equipamentos hospitalares, apresentadas a seguir.

\section{Condições que Provocam Problemas Osteomusculares}

\section{Levantamento e manuseio de pesos excessivos e repetitivos}

- Movimentar e transportar pacientes

- Retirar saco de roupa do "hamper"

- Transportar caixas com frascos de soro, monitores

- Movimentar respiradores

Levantamento e manuseio incorreto de cargas

- Levantar caixa de modo incorreto 
- Atividade com rotação exagerada do tronco

Solicitações extraordinárias e posturas inadequadas

- Preparar e movimentar bolsas para diálise

- Limpar sala de operação

- Puncionar veia

- Manuciar autoclave

Manutenção de uma postura por tempo prolongado

- Permanecer em pé por tempo prolongado ao lavar materiais

Outras condições

- Tensão emocional: assistir um paciente critico

- Trauma direto: segurar um paciente que está caindo

Fatores ergonômicos relacionados com o ambiente e equipamentos hospitalares

Espaço de trabalho

- Colocar paciente que utiliza cadeira de rodas em banheiro com espaço físico

- restrito

- Transportar um paciente em quarto sem espaço disponível

Superficie de trabalho

- Preparar medicações em bancada com altura inadequada

- Colher sangue em local improvisado e baixo

- Medir criança em local muito baixo

- Realizar procedimentos em camas e macas baixas

Limites de alcance

- Colocar em alturas elevadas ou delas retirar monitores, caixas de instrumental e soro

- Retirar prontuários e galões com líquidos de locais mais baixos, em armários

Equipamentos

- Falta de equipamentos auxiliares para transportar pacientes e objetos pesados

- Desnível entre as alturas da cama e da maca

- Cadeiras e macas com rodas de difícil movimentação

- Cama com grades e colchão d'água que dificultam a manipulação de pacientes

- Manivelas de camas de difícil movimentação

- Falta de travas em macas e camas 


\section{Orientações Ergonômicas Específicas}

Nesta parte da abordagem educativas são discutidos determinados aspectos ergonômicos relacionados com as atividades e posto de trabalho hospitalares.

Utilizando novamente fotos realizadas nas unidades do hospital, procura-se fornecer as orientações corretas ou as possíveis, dentro do contexto de trabalho encontrado.

É importante ressaltar que estas orientações são subsidiadas por publicações relacionadas especificamente com ergonomia (Armstrong et al., 1986, Bell, 1987, Chaffin, 1987, Dul, 1995, Grandjean, 1988, lida, 1990, Palmer, 1976, Raniere, 1989). Ao elaborar o conteúdo deste tópico, levou-se em consideração que grande parte das agressões à coluna vertebral em trabalhadores de enfermagem são causadas por traumas crônicos repetitivos, que envolvem muitos outros fatores, além da movimentação e transporte de pacientes, (Alexandre, 1993, Harber, 1987, Marziale, 1995, UNIVERSITY OF SURREY, 1986).

Nesse sentido, são abordados os seguintes tópicos:

\section{Orientações em Relação às Técnicas Adequadas de Levantamento, Manuseio e Transporte de Cargas}

- Avaliar a carga

- Respeitar os limites, segundo o sexo, freqüência e idade

- Manter um espaço livre para acesso à carga

- Norma regulamentadora ํㅜ 17-Ergonômica

- Obter condições seguras do solo e do trajeto a ser percorrido

- Posicionar os pês corretamente

- Segurar a carga usando totalmente as mãos

- Levantar cargas do chão com o dorso retificado e os joelhos dobrados

- Carregar a carga o mais próximo possível do corpo

- Evitar movimentos de torção em torno de eixo vertical do corpo

- Utilizar, sempre que possível, elementos e equipamentos auxiliares

- Participar periodicamente de programas de treinamento

\section{Orientações em Relação à Postura e Movimentos}

- Proporcionar variação de posições e atividades

- Observar a altura da bancada de trabalho de acordo com o tamanho do trabalhador e o tipo de serviço executado (bancadas para preparar medicações, medir crianças, coletar sangue)

- Manter altura de bancada ajustável, quando utilizada por diferentes pessoas (camas, macas) 
- Adaptar a altura da altura ou mesa à altura do assento

- Manter espaço suficiente para membros inferiores

- Colocar os pés alternadamente em um banquinho ao Ter que trabalhar em pé por tempo prolongado

- Evitar a inclinação do tronco mantendo os membros inferiores esticados (ex. ao retirar material esterilizado de armário)

- Evitaralcances excessivos

- Evitar o alongamento excessivo da coluna vertebral

- Armazenar objetos pesados dentro de uma amplitude de alturas próximas à cintura e os objetos leves em qualquer altura situada entre o joelho e a ombro (caixa de instrumental, monitores, roupas, soros, etc.)

- Colocar materiais em um nível que nunca ultrapasse a altura da cabeça

- Utilizar uma escadinha ao retirar objetos de partes altas de estantes já construídas (ex. pegar soros em armários)

\section{Orientações em Relação aos Equipamentos}

- Evitar a utilização de mobiliários equipamentos improvisados

- Atentar para uma manutenção adequada e periódica dos equipamentos hospitalares

- Modernizar o trabalho do pessoal de enfermagem pelo uso de equipamentos modernos, dando-se mais atenção ao aprovisionamento de auxílios mecânicos

- Avaliar equipamentos e mobiliários hospitalares, levando em conta fatores ergonômicos

\section{Orientações Diversas}

Considerando que os estudos sobre as lesões músculo-esqueléticas têm gerado inúmeras e que geralmente abrangem múltiplas variáveis com complexo interrelacionamento, cada vez mais buscam-se novas abordagens para enfrentar o problema. Nesse sentido, surgem programas com estratégias que caminham em direção a uma abordagem holística (Hellsing, et al., 1993, Linton, 1989, Venning, 1988).

Levando em consideração esses dados e tendo como subsídio estudos e recomendações de autores (Cailliet, 1979, Knoplick, 1986, La Freniere, 1981) que se preocupam com a questão das dores osteomusculares, peocura-se nessa fase fornecer as seguintes orientações:

- Importância da prática regular de exercícios físicos

- Esportes e sua interferência no sistema osteomuscular

- Importância do relaxamento 
- Posições, colchões e travesseiros mais adequados para dormir

- Interferência da tensão emocional e estilo de vida sobre as dores nas costas

- Considerações sobre o tratamento e medidas para aliviar as dores

\section{Movimentação e transporte de pacientes}

Os procedimentos que envolvem a movimentação e transporte de pacientes são considerados os mais penosos para os trabalhadores de enfermagem. Estudiosos da questão defendem que o ensino desses procedimentos deve ser complementando com uma avaliação do local de trabalho ed e alternativas para torná-los menos prejudicais. (Owen, 1993, UNIVERSITY OF SURREY, 1986).

Considerando tais aspectos, dividiu-se esta última fase em quatro partes:

\section{Avaliação das Condições e Preparo do Paciente}

Neste tópico descrevem-se os principais cuidados e orientações que devem ser ministrados aos pacientes. Inicialmente é enfatizada a importância de uma avaliação das condições físicas da pessoa que será movimentada, de sua capacidade em colaborar, bem como a observação da presença de soros, sondas e outros equipamentos conectados. Faz-se a orientação para um planejamento cuidadoso do procedimento e explicação ao paciente do modo como se pretende movê-lo, como pode cooperar, para onde será encaminhado e qual o motivo da locomoção. É importante salientar que o paciente deve ser orientado a ajudar sempre que for possível. Outro ponto comentado é que a movimentação e transporte de obesos precisam ser minuciosamente avaliados e planejados.

\section{Preparo do Ambiente e Equipamentos}

Considerando que determinados aspectos do posto de trabalho podem prejudicar os procedimentos de movimentação e transporte, aborda-se nessa parte os principais cuidados que necessitam ser considerados:

- Observar o espaço físico

- Verificar o local e remover os obstáculos

- Observar a disposição do mobiliário

- Obter condições seguras com relação ao piso

- Colocar o suporte de soro ao lado da cama quando necessário

- Elevar ao abaixar a altura da cama para ficar no mesmo nível da cama

- Travar a altura da cama, maca e cadeira de ou solicitar auxílio adicional

- Adaptar a altura da cama ao profissional e ao tipo de procedimento que será realizado

\section{Preparo da Equipe de Enfermagem}

Nesta fase são descritas algumas orientações específicas para o pessoal de enfermagem, principalmente relacionadas com os princípios básicos de mecânica corporal, que devem ser utilizados durante a manipulação de pacientes:

- Deixar os pés afastados e totalmente apoiados no chão

- Manter as costas eretas

- Usar o peso corporal como um contrapeso ao do paciente 
- Flexionar os joelhos ao invés de curvar a coluna

- Abaixar a cabeceira da cama ao mover um paciente para cima

- Utilizar movimentos sincrônicos

- Trabalhar o mais próximo possível do corpo do paciente, que deverá erguido ou movido

- Usar uniforme e sapatos apropriados

- Utilizar elementos auxiliares tais com: barra tipo trapézio no leito, cinto de transfer6encia, plástico antiderrapante para os pés, rolo plástico facilitador de movimentos, prancha de transferência, auxílios mecânicos, entre outros.

\section{Movimentação de Pacientes}

Visa demonstrar através de "slides" a forma adequada de:

- Movimentar o paciente, que não po.de colaborar, para a cabeceira da cama

- Movimentar o paciente, que auxilia, para a cabeceira da cama

- Aproximar o paciente para um dos lados da cama

- Colocar o paciente em decúbito lateral

- Sentar o paciente na beira da cama

\section{Transporte de Pacientes}

Tem por objetivo demostrar, por meio de uma seqüência de "slides", o modo correto de transportar o paciente:

- Do leito para a cama, com auxílio de um lençol

- Do leito para a cadeira de rodas (quando o paciente auxilia)

- Do leito para a cadeira de rodas (paciente incapacitado)

\section{CONSIDERAÇÕES FINAIS}

Este trabalho apresenta a parte educativa de um programa mais amplo, cujo objetivo é contribuir para a redução de lesões no sistema músculo-esquelético de trabalhadores de enfermagem. É uma abordagem inovadora, uma vez que foi desenvolvida a partir da análise de posto de trabalho e das atividades executadas por esse pessoal, levando em consideração um enfoque ergonômico.

\section{REFERÊNCIASBIBLIOGRÁFCAS}

1. ALEXANDRE, N.M.C. Avaliação de determinados aspectos ergonômicos no transporte de pacientes. Ribeirão Preto, 1987. 114p. Dissertação (Mestrado) Escola de Enfermagem de Ribeirão Preto, Universidade de São Paulo.

2. ALEXANDRE, N.M.C. et aí. Aspectos ergonômicos e posturais em centro de material. Rev.Esc.Enferm. USP, v. 26, n. 1, p. 87-94, 1992.

3. ALEXANDRE, N.M.C. Contribuição ao estudo das cervicodorsolombalgias em profissionais de enfermagem. Ribeirão Preto, 1993. 186p. Tese (Doutorado) Escola de Enfermagem de Ribeirão Preto, Universidade de São Paulo. 
4. ARMSTRONG, T.J. et aí. Repetitive trauma disorders: job evaluation and design. Human Factors, v. 28, n. 3, p. 325-36, 1986.

5. BARREIRA, T.H.C. Um enfoque ergonômico para as posturas de trabalho. Rev. Bras. Saúde Ocup., v 17, n. 67, p. 61-71, 1989.

6. BELL, F. Ergonomic aspects of equipament. Int. J. Nurs. Stud., v. 24, n. 4,p. 331-7, 1987. 7. BURGMEIER, A.C. et aí. Les lombalgies in milieu hospitalar: aspects épidémiologiques et rôle des divers facteurs de risque. Rev. Epidém. et Santé Publ.,v. 36, n. 2, p. 128-37,1988.

8. CAILLIET, R. Sindromes dolorosas. Lombalgias. São Paulo: Manole, 1979.

9. CHAFFIN, D.B. Occupational biomechanics - a basis for workplace design to prevent musculoskeletal injuries. Ergonomics, v. 30, n. 2, p. 321-9,1987.

10. COHEN-MANSFIELD, J. et aí. Nursing staft back injuries. MOHN J., v. 44, n. 1, p. 9-17, 1996.

11. DAWS, J. Lifting and moving patients. A revision training programme. Nurs. Times, v. 77, n. 48, p. 2067-9,1981.

12. DE ROSA, C.P. ; PORTERFIELD, J.A A physical therapy model fbr the treatment of low back pam. Phvsical Therapv, v. 72, n. 4, p. 261-9,1992.

13. DONCHIN, M. et aí. Secondary prevention of $10 \mathrm{w}$ back pam. Spine, v. 15, n. 12 , p. $1317-20,1990$.

14. DUL, J. WEERDMEESTER, B. Ergonomia prática. São Paulo: Ed. Edgard Blucher, 1995.

15. ENGELS, J. A et aí. Work related risk factors for musculoskeletal complaints in the nursing profession: results of a questionnaire survey. Occup. Environ. Med., v. 53, p. 636-41, 1996.

16. GARG, A.; MOORE, J.S. Epidemiology of low back pam in industry. Occup. Med., v. 7, n. 4, p. 593-608, 1992.

17. GRANDJEAN, E. Fitting the task to the man. $4^{\mathrm{a}}$ ed. London: Taylor \& Francis, 1988.

18. GUO, H.R. et aí. Back pam among workers in the United States: nationai estimates and workers at high risk. Am. J. Ind. Med., v. 28, n. 5, p. 591-602, 1995.

19. HALL, 5. J. Biomecánica básica. Rio de Janeiro: Guanabara Koogan, 1993.

20. HARBER, P. et aí. Occupational low back pam in hospital nurses. J. Occup. Med., V. 27, n. 7, p. 518-24, 1985.

21. HARBER, P. et ai. Importance of non-patient transfer activities in nursing - related back pam. J. Occup. Med., v. 29, n. 12, p. 967-70,1987.

22. HELLSING, A L. et ai. Ergonomic education for nursing students. Int. J. Nurs. Stud., v. 30, n. 6, p. 499-510, 1993.

23. HILL. T. Equipped for the job. Nurs. Times, v. 79, n. 27, p. 25-7, 1983.

24. IIDA, 1. Erqonomia: proieto e produção. São Paulo: Edgard Biucher, 1990.

25. JENSEN, C. R.; SCHULTZ, G. W. Applied Kinesiologv. New York: McGraw-Hill, 1977. 
26. KAPANDJI, 1. A. Fisiologia articular: esquemas comentados de mecánica humana. São Paulo: Manole, 1979.

27. KELLET, K.M. et aí. Effects of an exercise program on sick leave due to back pam. Psvsical Therapv, v. 71, n. 4, p. 283-93, 1991.

28. KENDALL, H.O. et aí. Posture and pam. New York: Krieger, 1977.

29. KNIBBE, J.J. ; FRI ELE, R.D. Prevalence of back pam and characteristics of the physical workload of community nurses. Ergonomics, v. 39, n. 2, p. 186-98, 1996. 30. KNOPLICK, J. Enfermidades da coluna vertebral. 2. ed. São Paulo: Panamed, 1986.

31. LA FRENIERE, J.G. El paciente com lumbalgia. Barcelona: Toray-masson, 1981.

32. LEIGHTON, D.J. ; REILLY, T. Epidemiological aspects of back pam: the incidence and prevalence of back pam in nurses compared to the general population. Occup. Med., v. 45, n. 5, p. 263 -7, 1995.

33. LENIHAN, J.M.A Desing in health care. Nurs. Mirror, v. 156, v. 26, p. 25-6, 1983.

34. LEONARD, S.A The role of exercise and posture in preventing $10 \mathrm{w}$ back injury. AAOHNJ., v. 38, n. 7, p. 318-22, 1990.

35. LINTON, S.J. et. al. The secundary prevention of $10 \mathrm{w}$ back pam. Pam, v. 36, n. 2, p. 197-207, 1989.

36. MAGNI, G. et al. Prospective study on the relationship between depressive symptoms and chronic musculoskeletal pam. Pam, v. 56, n. 3, p. 289-97, 1994.

37. MARZIALE, M.H. P. Condicões ergonômicas da situacáo de trabalho do pessoal de enfermagem em uma unidade de internação hospitalar. Ribeirão Preto, 1995. 163p. Tese (Doutorado) - Escola de Enfermagem de Ribeirão Preto, Universidade de São Paulo.

38. MOFFETT, J.A K et ai. A controlled, prospective study to evaluate the eftectiveness of a back school in relief of chronic 10w back pam. Spine, v. 11, n. 2, p. 120-2,1986.

39. OWEN, B. D. ; GARG, A Back stress isn't part of the job. Amer. J. Nurs., v. 93, n. 2, p. 48-51, 1993.

40. PALMER, C. Ergonomia. Rio de Janeiro: Fundação Getúlio Vargas, 1976.

41. PARNIAPOUR, M. et ai. Environmentaliy induced disorders of the musculoskeletal system Med. Clin. North Am., v. 74, n. 2 , p. 347-59, 1990.

42. PATENAUDE, S.S.; SOMMER, M. A. Low back pam. AORN J., n. 3, v. 46,1987.

43. RAISTRICK, A Nurses with back pam - can the problem be prevented? Nurs. Times, v. 77, n. 14 , p. 853-6,1981.

44. RANIERE, T.M. Prevention of cumulative trauma injuries. AAOHN J., v. 337, n. 6, p. 221-4,1989.

45. REILLY, K et ai. Diferences between a supervised and independent strength and conditioning program with chronic $10 \mathrm{w}$ back syndromes. J. Occup. Med., v. 31, n. 6 , p. 547-50,1989.

46. ROSS, P. Ergonomic hazards in the workplace. AAOHN J., v. 42, n. 4, p171-6, 1994. 47. SCHOLEY, M. Patient handling skills. Nurs. Times, v.80, n. 26, p. 25-7, 1984. 
48. STUBBS, D.A et ai. Back pam in the nursing profession. Ergonomics, v. 26, n. 8 , p. 755-65, 1983.

49. SVENSON, H. ; ANDERSON, G.B.J. The relationship of $10 \mathrm{w}$ back pam, work history, work environment and stress. Spine, v. 14, n. 5, p. 517-22, 1989.

50. THOMPSON, C. Leaming to lift. Nurs. Times, v. 83, n. 15, p. 34-5, 1987.

51. TROUP, J.D.G. ; RAUHALA, H.H. Ergonomics and training. Int. J. Nurs. Stud., V. 24, n. 4, p. 325-30, 1987.

52. UNIVERSITY OF SURREY. Ergonomics Research Unit. Back pain in nurses: summary and recommendations. Guildford, 1986.

53. VENNING, P.J. Back injury prevention among nursing personel. AAOHN J., v. 36, n. 8, p. 327-33, 1988.

54. VIDEMAN, T. et ai. Low backpam in nurses and some loading factors of work. Spine, V. 9, n. 4, p. 400-4, 1984.

55. VIDEMAN, T. et ai. Patient handling skill, back injuries and back pam. Spine, v. 14, n. 2, p. 145-55, 1989.

56. WESTGMRD, R.H. ; MRAS, A The eftect of improved workplace design on the development of work related musculoskeletal illness. Applied Ergonomics, v. 16, n. 2, p. 91-7, 1985.

57. WORLD HEALTH ORGANIZATION. Identification and control of work-related diseases. Geneva: 1985. (Techn. Rep. Ser. 7144).

58. YASSI, A et ai. The epidemiology of back injuries in nurses at a large Canadian tertiary care hospital: implications for prevention. Occup. Med., v. 45, n. 4, p. 21520, 1995.

59. YELIN, E.; CALLAHAN, L.F. The economic cost and social and psychological impact of musculoskeletal conditions. Arthritis Rheum., v. 38, n. 10, p. 1351-62, 1995. 